\title{
SIMULATION OF MOTION OF A MOBILE ROBOT ON UNEVEN TERRAIN
}

\author{
Przemyseaw Sperzyński, Jacek BaŁchanowski, Antoni Gronowicz \\ Wroctaw University of Science and Technology, Faculty of Mechanical Engineering, Wroclaw, Poland \\ e-mail: przemyslaw.sperzynski@pwr.edu.pl
}

\begin{abstract}
In the paper, a kinematical model of the robot moving on uneven terrain has been presented. Configuration of the robot placed on uneven terrain is described, presenting the redundant set of variables that create the state of the robot. Inverse kinematics tasks have been defined, which as a result of various assumptions, gives the robot configuration. To solve the kinematics tasks, adequate closed-loop equations have been defined. Velocity equations of the point of the wheel contact with the ground have been presented with respect to the velocity of the platform itself and velocities of the robot actuators. Assuming constraints of the wheel movement causing a no-slip condition, dependencies between those velocities have been determined. This allowed one to determine velocities of the robot drives, so that the platform moves in the way specified by the user.
\end{abstract}

Keywords: wheeled mobile robot, contact analysis, inverse kinematics computation

\section{Introduction}

Mobile robots are a subject of research around the world. The main task performed by such robots is broadly understood as transport of goods and, sometimes, people as well. Numerous examples can be found of wheeled tracked robots as well as walking or even flying robots.

In the recent years, most often mobile platforms are used to automate warehouses or production facilities, where moving items from a place to place is the most important task. The main type of locomotion, in this case, are wheeled robots (Avilés et al., 2018; Bonnafous et al., 2018; Wise et al., 2018). This is the most effective way of travelling in a flat field. Sometimes, however, obstacles encountered by the robot do not allow further movement, and the only way to overcome the obstacle is the detour.

Inspection is another example of a task for mobile robots. Here, commonly, hybrid tracked chassis systems are used (Novák et al., 2018). It allows one to overcome obstacles such as stairs. Tracked robots in comparison to wheeled ones move significantly slower.

A more universal way of locomotion on terrain with unevenness is gait. Walking bipeds (www.bostondynamics.com/atlas), quadruped robots (Grand et al., 2010), and also those made with more limbs (Townsend, 2011) can be found in the literature.

The combination of wheel suspension and walking robots gives a hybrid locomotion system. Wheeled-legged robots have the advantages of both types of locomotion. They can move quickly on wheels, driving on a flat ground. In addition, when such a robot encounters an obstacle that prevents further driving, it can overcome it by walking. Furthermore, an active wheel suspension allows levelling of the platform. Wheeled-legged robots are a subject of research in many research centres all around the world (Bałchanowski, 2016; Grand et al., 2010; Ylönen and Halme, 2002).

At Wrocław University of Technology, a prototype of a wheeled-legged robot (Bałchanowski, 2016; Sperzyński and Szrek, 2013) was designed and built. It has a specific wheel suspension system which simplifies the levelling task. Simulation of robot movement has been a topic of 
the research for many years (Bałchanowski, 2016; Chakraborty and Ghosal, 2004; Sperzyński and Gronowicz, 2017). The paper presents the kinematical model of this mobile robot, allowing motion planning on uneven terrain.

\section{System description}

The described wheeled-legged robot in (Bałchanowski, 2016) consists of four identical limbs placed along the platform (Fig. 1a), where each of them ends with a driven wheel. The limb has mobility equal to four. Two linear drives $s_{1}$ and $s_{2}$ are used to manipulate the position of the wheel along the mobile platform. They are used for levelling of the platform or for walking (Fig. 1b). The wheel position manipulation mechanism is planar, allowing the wheel to be displaced only in the plane of the limb frame at $A$, as shown in Fig. 1b. The next two angular actuators $\theta_{3}$ and $\theta_{4}$ are used to steer and drive the wheel. The axes of rotation of both drives go through the center of the wheel - point $F$ and, therefore, do not affect its position.

(a)

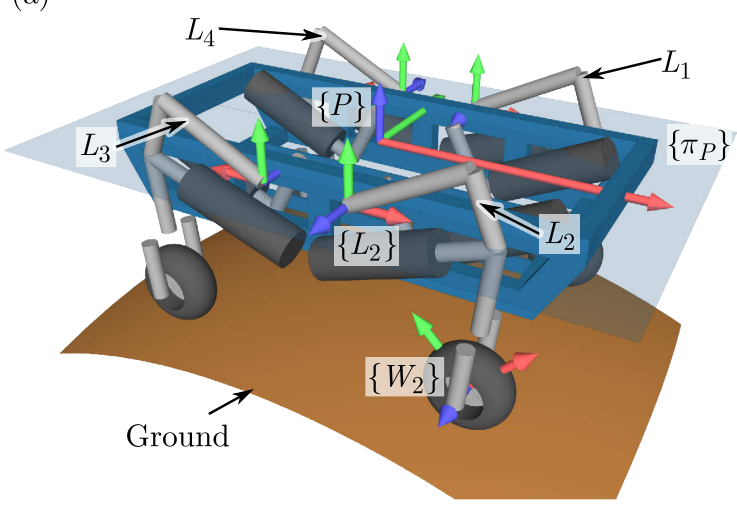

(b)

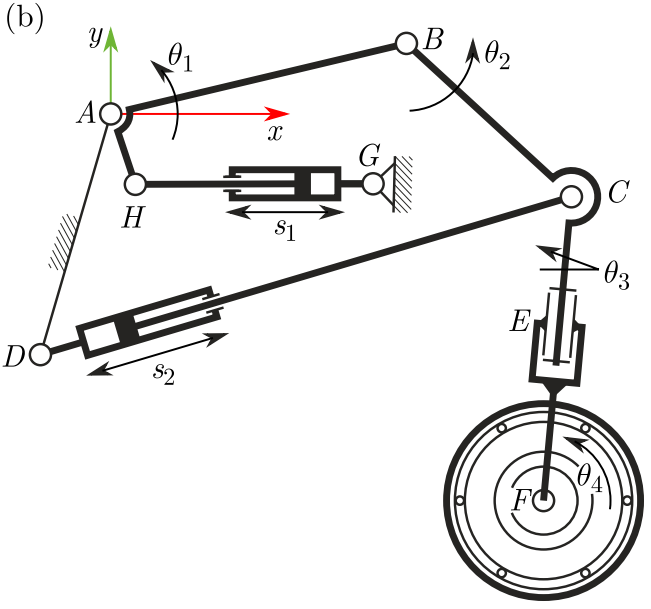

Fig. 1. (a) Described wheeled-legged robot, (b) kinematic scheme of the robot limb

(a)

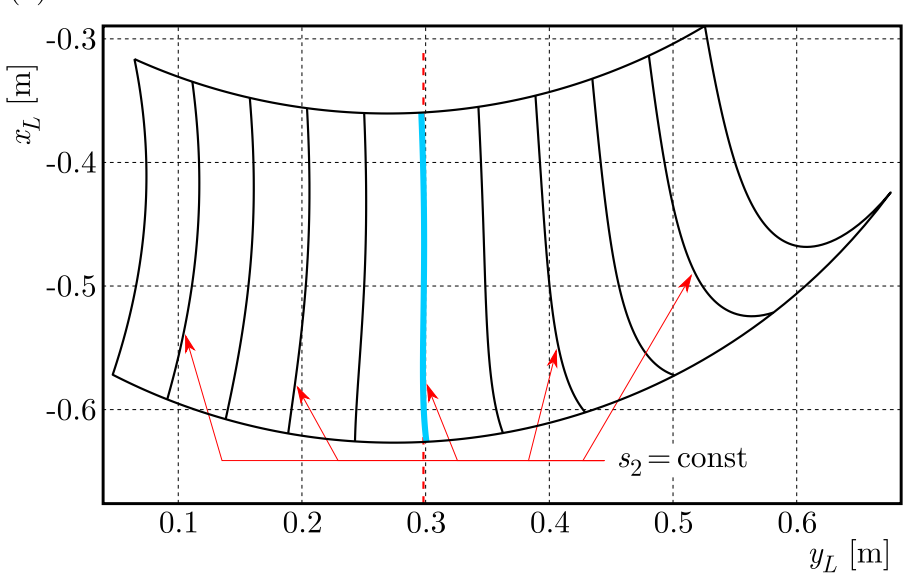

(b)

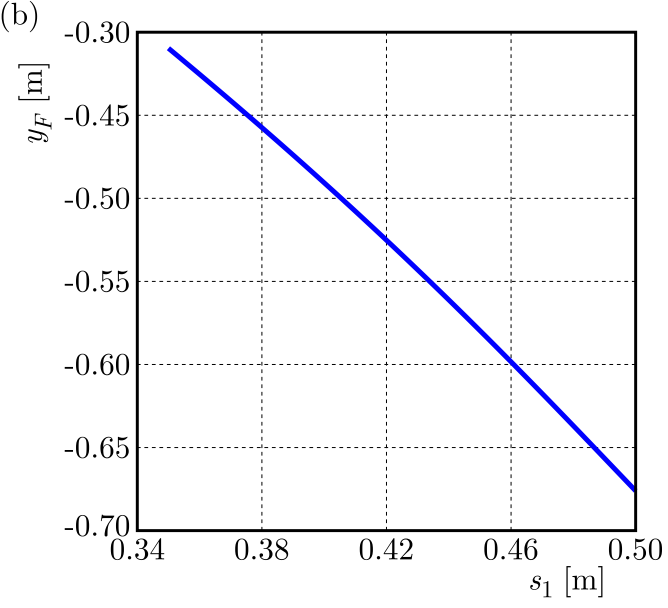

Fig. 2. Kinematic characteristics used to level the robot platform, (a) workspace of the robot limb, (b) wheel level characteristics as a function of the linear actuator

The dimensions of the limb were selected in such a way as to obtain a sufficiently large workspace (Fig. 2a) (Sperzyński and Szrek, 2013; Bałchanowski, 2016). The $s_{1}$ actuator is responsible for changing the value of the angle $\theta_{1}$. It is mainly used to change the vertical position of the 
wheel. Figure $2 \mathrm{a}$ shows the trajectory of the center of the wheel when the strokes of the $s_{2}$ linear actuators are fixed and the drive $s_{1}$ changes over its entire range. Parameters of the limb were selected so as to obtain a rectilinear trajectory of the center of the wheel for fixed particular actuator positions $s_{2}=$ const. As can be seen, being closer the center of the workspace, the trajectory of the point $F$ approaches a straight line. The characteristics of the vertical position of the wheel, depending on length of the drive $y_{F}\left(s_{2}\right)$, are similar to linear ones (Fig. $2 \mathrm{~b}$ ). This characteristic corresponds to the trajectory marked in blue in Fig. 2a. This fact simplifies the platform levelling control system, because for this purpose one drive per limb is used. The second linear actuator $s_{2}$ is only responsible for changing the angle $\theta_{2}$. The assumption is that it should be used to perform gait, when it is necessary to move the wheel horizontally. Figure 1b shows that the change in angle $\theta_{2}$, with a fixed stroke of $s_{1}$, leads the point $F$ along the arc with the center at point $B$ and radius equal to $B F$.

The kinematics of the walking robot are described in a similar way as in the case of parallel manipulators. The effector is the robot platform, and the ground on which the robot moves is the base of the mechanism. The effector is connected to the base of the mechanism by means of several kinematic chains - the robot limbs. The location and orientation of the mobile platform defines the set of $\mathbf{X}$ task variables. Subsequently, each kinematic chain introduces $\boldsymbol{\theta}_{i}$ joint variables that determine configuration of the limb. In the case of wheeled robots, the connection of the robot limb to the base is a nonholonomic constraint and, therefore, will be described by additional contact variables $c_{i}$ excluded from the joint variable space.

The robot description, platform configuration in space and its limbs as well as contact conditions with the ground create a set of variables hereinafter referred to as the state of the robot $\mathbf{q}$

$$
\mathbf{q}=[\mathbf{X}, \boldsymbol{\theta}, \mathbf{c}]^{\mathrm{T}} \quad \mathbf{X}=\left[\mathbf{p}_{P}, \boldsymbol{\varphi}_{P}\right]^{\mathrm{T}} \quad \boldsymbol{\theta}=\left[\boldsymbol{\theta}_{1}, \ldots, \boldsymbol{\theta}_{n_{L}}\right]^{\mathrm{T}} \quad \mathbf{c}=\left[\mathbf{c}_{1}, \ldots, \mathbf{c}_{n_{C}}\right]^{\mathrm{T}}
$$

where: $\mathbf{p}_{P}$ it is the location of the platform $\left(x_{P}, y_{P}, z_{P}\right), \boldsymbol{\varphi}_{P}$ - platform orientation in space defined by three, yaw, pitch and roll angles $\left(\varphi_{Y P}, \varphi_{P P}, \varphi_{R P}\right), n_{L}-$ amount of the robot limbs, $n_{C}$ - number of wheels in contact with the ground.

On the robot platform, the $\{P\}$ frame has been defined. It is placed in the middle of the platform (Fig. 1a). The $\widehat{\mathbf{x}}_{P}$ axis is positioned along the platform, and its direction indicates the front of the robot. The axis $\widehat{\mathbf{y}}_{P}$ is set towards the left side of the platform (indicates lest limbs). These axes define the platform plane $\pi_{P}$. The $\mathbf{X}$ vector contains location variables and platform orientation angles that describe the $\{P\}$ frame relative to the global frame $\{G\}$

$$
\begin{array}{rlr}
\{P\}: & { }^{G} \mathbf{A}_{P} & =\left[\begin{array}{cc}
{ }^{G} \mathbf{R}_{P} & { }^{G} \mathbf{p}_{P} \\
\mathbf{0} & \mathbf{1}
\end{array}\right] \quad{ }^{G} \mathbf{P}_{P}=\left[x_{P}, y_{P}, z_{P}\right]^{\mathrm{T}} \\
{ }^{G} \mathbf{R}_{P} & =\mathbf{R}_{Z}\left(\varphi_{Y P}\right) \mathbf{R}_{Y}\left(\varphi_{P P}\right) \mathbf{R}_{X}\left(\varphi_{R P}\right)
\end{array}
$$

where ${ }^{G} \mathbf{A}_{P}$ - transformation matrix of the frame $\{G\}$ to the platform frame $\{P\}$ consisting of the rotation matrix ${ }^{G} \mathbf{R}_{P}$ and the location vector ${ }^{G} \mathbf{p}_{P}, \mathbf{R}_{i}(a)$ - matrix of rotation around the $i$-th axis by an angle of the value of $a$.

On the $\pi_{P}$ plane, there are local coordinate systems $\left\{L_{i}\right\}$ of the limbs. and they are located symmetrically in relation to the axis of the platform system $\{P\}$. The orientation of the limb systems is set perpendicular to the plane of the $\pi_{P}$ platform, so that the axes $\widehat{\mathbf{z}}_{L_{i}}$ lie in this plane. The limbs are numbered in accordance with Fig. 1a. At $F$, the center of the wheel, the $\left\{W_{i}\right\}$ wheel local frame, is defined. The parameter vector $\boldsymbol{\theta}_{i}$ contains variables describing configuration of the limb and transformation ${ }^{L_{i}} \mathbf{A}_{W_{i}}$ the wheel system $\left\{W_{i}\right\}$, from its base $\left\{L_{i}\right\}$

$$
\boldsymbol{\theta}_{i}=\left[s_{1 i}, s_{2 i}, \theta_{3 i}, \theta_{4 i}\right]^{\mathrm{T}} \Longrightarrow{ }^{L_{i}} \mathbf{A}_{W_{i}}
$$


It was further assumed that the wheel contact with the ground is a point contact (class $\mathrm{V}$ kinematic pair), and thus it should be described with five parameters. They were named as $\mathbf{c}_{i}$, and for each wheel in contact they are variables

$$
\mathbf{c}_{i}=\left[c_{1 i}, \ldots, c_{5 i}\right]^{\mathrm{T}}
$$

In order to describe contact variables, solid models of the ground and wheels must be defined. By performing dimensional analysis, the number of closed-loop equations needed to describe the robot was determined. For one limb in contact with the ground $\left(n_{L}=n_{C}=1\right)$, the dimension of the variable vector is equal to $\operatorname{dim} \mathbf{q}=15$. The mobility of a single kinematic chain of the limb connecting the platform to the ground is ${ }^{P+} L_{i} W_{T}=9$. The difference shows that six independent loop equations should be defined for each of the robot limbs in contact with the ground in the form of

$$
\boldsymbol{\phi}_{i}\left(\mathbf{X}, \boldsymbol{\theta}_{i}, \mathbf{c}_{i}\right)=\mathbf{0} \quad \text { where } \quad \boldsymbol{\phi}_{i}=\left[\phi_{1 i}, \ldots, \phi_{6 i}\right]^{\mathrm{T}}
$$

The inverse kinematics task is to determine the configuration of kinematic chains (variables $\boldsymbol{\theta}_{i}$ and $\mathbf{c}_{i}$ ) at a given location and orientation of the platform - variables $\mathbf{X}$. This task can be solved for each limb independently. In the case of a mobile robot, this task is to lower the limbs until the wheels are in contact with the ground. Analysing the size of the variable vector for one limb, the difference between the number of equations and the number of variables is $\operatorname{dim} \boldsymbol{\theta}_{i}+\operatorname{dim} \mathbf{c}_{i}-\operatorname{dim} \boldsymbol{\phi}_{i}=4+5-6=3$, which means that to solve the task, three variables from $\boldsymbol{\theta}_{i}$ has to be set. It has been assumed that $\mathbf{c}_{i}$ contact variables are determined by solving the kinematics task at a particular drive position.

It was assumed that the $s_{2}$ actuators and wheel orientation $-\theta_{3}$ and $\theta_{4}$ of each limb were fixed in a particular position. Such a stroke $s_{1}$ of the linear actuator and all contact variables $c_{i}$ should be found. This ensures the point contact of the wheel with the ground (Fig. 3a).

(a)

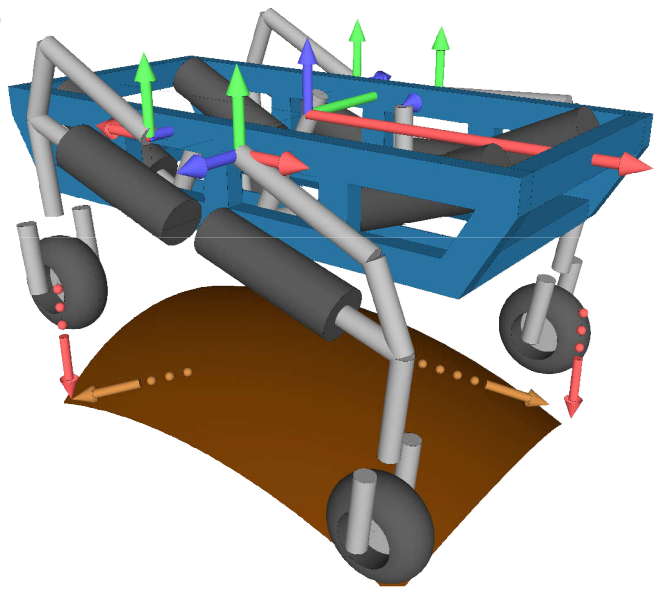

(b)

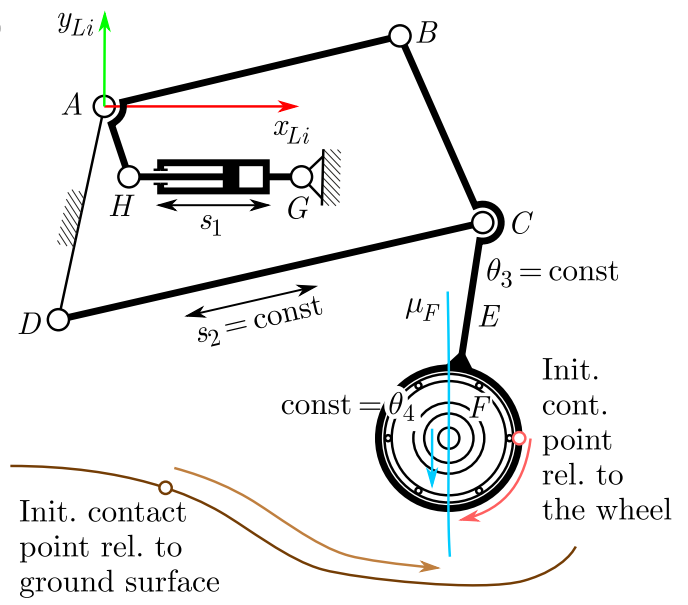

Fig. 3. The way of solving the inverse kinematics task, (a) determination of limb configuration to be in contact with the ground, (b) kinematic scheme of the robot limb

In Fig. 3b, it is shown how for the assumed initial points on the circumference of the wheel and on the surface of the ground the wheel is lowered until the contact constraints are met (2.1). In order to solve the inverse task for the presented robot, the procedure should be repeated for each limb separately (Fig. 3a).

\subsection{Point contact models of the wheel surface and the ground}

The terrain was modelled as a surface element, described parametrically, depending on two parameters $u_{G}$ and $v_{G}$. The surface will be defined in the $\{G\}$ ground coordinate system. The location of any point $S$ belonging to the ground surface is expressed as follows 


$$
{ }^{G} \mathbf{p}_{S}=\mathbf{f}_{G}\left(u_{G}, v_{G}\right)=\left[\begin{array}{c}
f_{x G}\left(u_{G}, v_{G}\right) \\
f_{y G}\left(u_{G}, v_{G}\right) \\
f_{z G}\left(u_{G}, v_{G}\right)
\end{array}\right] \quad \mathbf{f}_{G}: \mathbb{R}^{2} \rightarrow \mathbb{R}^{3}
$$

Figure 4 a shows an example of a terrain surface. Respectively, the local coordinate system $\{C G i\}$ located at the point $S$ can be defined given by the transformation matrix ${ }^{G} \mathbf{A}_{C G i}$, such that its axis ${ }^{G} \widehat{\mathbf{x}}_{C G i}$ and ${ }^{G} \widehat{\mathbf{y}}_{C G i}$ defines the plane tangent to the surface at this point. The surfaces will be defined as convex hull, i.e. the unit vector ${ }^{G_{\mathbf{z}}}{ }_{C G i}$ will be pointed outside the body of the element (Fig. 4a). The ${ }^{G} \mathbf{A}_{C G i}$ transformation matrix, defined using the $u_{G}$ and $v_{G}$ parameters, is expressed as follows

$$
{ }^{G} \mathbf{A}_{C G i}=\left[\begin{array}{cc}
{ }^{G} \mathbf{R}_{C G i} & { }^{G} \mathbf{p}_{C G i} \\
\mathbf{0} & \mathbf{1}
\end{array}\right]
$$

where

$$
{ }^{G} \mathbf{R}_{C G i}=\left[{ }^{G} \widehat{\mathbf{x}}_{C G i},{ }^{G} \widehat{\mathbf{y}}_{C G i},{ }^{G} \widehat{\mathbf{z}}_{C G i}\right] \quad{ }^{G} \mathbf{p}_{C G i}=\mathbf{f}_{G}
$$

(a)

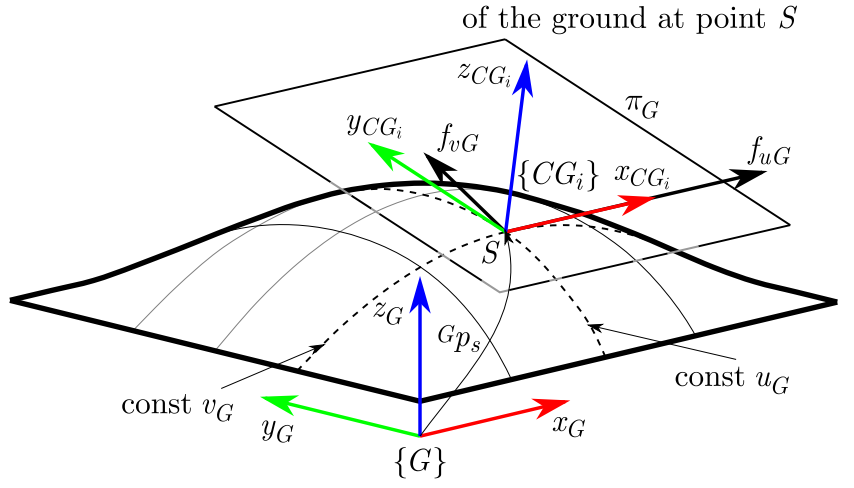

(b)

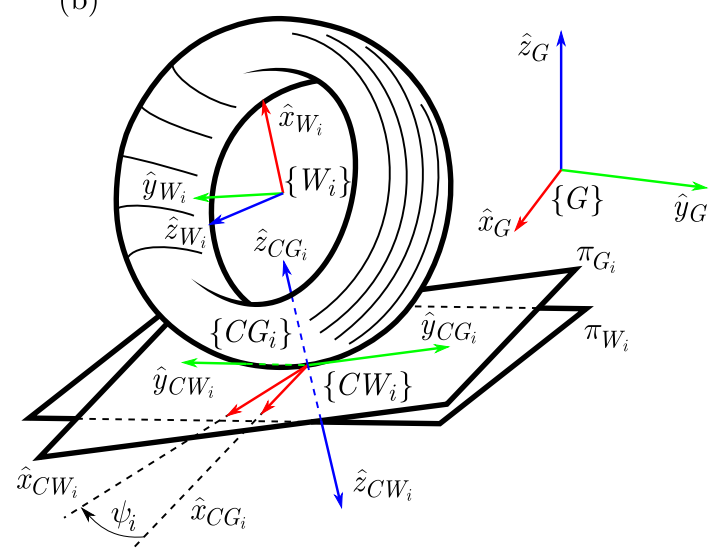

Fig. 4. (a) Definition of the coordinate system defining the tangent plane to the ground surface,

(b) contact conditions of two surfaces

At the point $S$, two vectors $\mathbf{f}_{u G}$ and $\mathbf{f}_{v G}$, tangent to the parametric surface, can be defined and will define the $\pi_{G}$ tangent plane to the ground surface

$$
\mathbf{f}_{u G}=\frac{d f_{G}}{d u_{G}} \quad \mathbf{f}_{v G}=\frac{d f_{G}}{d v_{G}}
$$

Using the vectors that span the plane $\pi_{G}$, the axis ${ }^{G} \widehat{\mathbf{x}}_{C G i}$ is defined as

$$
G_{\widehat{\mathbf{x}}_{C G i}}=\frac{\mathbf{f}_{u G}}{l_{f u G}} \quad l_{f u G}=\sqrt{\mathbf{f}_{u G}^{\mathrm{T}} \cdot \mathbf{f}_{u G}}
$$

The normal vector ${ }^{G} \widehat{\mathbf{z}}_{C G i}$ to the $\pi G i$ plane is defined as the product of the tangent vectors $\mathbf{f}_{u G}$ and $\mathbf{f}_{v G}$

$$
G_{\widehat{\mathbf{z}}_{C G i}}=\frac{1}{l_{n G}}\left(\mathbf{f}_{u G} \times \mathbf{f}_{v G}\right) \quad l_{n G}=\sqrt{\left(\mathbf{f}_{u G}^{\mathrm{T}} \cdot \mathbf{f}_{u G}\right) \cdot\left(\mathbf{f}_{v G}^{\mathrm{T}} \cdot \mathbf{f}_{v G}\right)-\left(\mathbf{f}_{u G}^{\mathrm{T}} \cdot \mathbf{f}_{v G}\right)^{2}}
$$

The orientation of the ${ }^{G} \widehat{\mathbf{y}}_{C G i}$ axis is given by the vector product

$$
G_{\widehat{\mathbf{y}}_{C G i}}=\left(G_{\widehat{\mathbf{z}}_{C G i}} \times{ }_{\widehat{\mathbf{x}}_{C G i}}\right)=\frac{l_{f u G}}{l_{n G}} \mathbf{f}_{v G}-\frac{\mathbf{f}_{u G}^{\mathrm{T}} \cdot \mathbf{f}_{v G}}{l_{f u G} l_{n G}} \mathbf{f}_{u G}
$$


which fully defines the $\{C G i\}$ coordinate system. Similarly, in the case of the wheel, its surface is defined by the parameters $u_{W_{i}}$ and $v_{W_{i}}$ via the wheel shape function $\mathbf{f}_{W_{i}}$ (analogously to surface definition (2.2)). The shape of the tire is defined in the wheel local coordinate system $\left\{W_{i}\right\}$. The $\{C W i\}$ system, which describes the contact point relative to the wheel, as in the case of the ground, is defined parametrically according to relationship (2.3). The model of point contact of the wheel with the ground forces the $\pi_{G}$ and $\pi_{W_{i}}$ planes (tangent to both bodies at the point of contact) to be coplanar. The $\widehat{\mathbf{z}}_{C G i}$ and $\widehat{\mathbf{z}}_{C W i}$ axes will then be opposite (collinear), and the local contact systems $\{C G i\}$ and $\{C W i\}$ will be rotated relative to each other by a certain angle $\Psi_{i}$ (Fig. 4b). The transformation matrix from the global system to the wheel contact system (between bodies in contact) is defined as follows

$$
{ }^{G} \mathbf{A}_{C W i}={ }^{G} \mathbf{A}_{W_{i}} \cdot{ }^{W_{i}} \mathbf{A}_{C W i}={ }^{G} \mathbf{A}_{C G i} \cdot{ }^{C G i} \mathbf{A}_{C W i}
$$

where the contact frames are placed at the same point, so ${ }^{C G i} \mathbf{p}_{C W i}=\mathbf{0}$, and the rotation matrix between these systems is given by the dot product

$$
{ }^{C G i} \mathbf{R}_{C W i}=\mathbf{R}_{Z}\left(\Psi_{i}\right) \mathbf{R}_{X}(\pi)
$$

Dependencies (2.4) can be transformed into a form that gives the location and orientation of the wheel relative to the ground by describing the wheel contact with the ground

$$
{ }^{G} \mathbf{A}_{W_{i}}={ }^{G} \mathbf{A}_{C G i}\left(u_{G_{i}}, v_{G_{i}}\right) \cdot{ }^{C G i} \mathbf{A}_{C W i}\left(\Psi_{i}\right) \cdot{ }^{C W i} \mathbf{A}_{W_{i}}\left(u_{W_{i}}, v_{W_{i}}\right)
$$

The contact is, therefore, defined by a set of five contact parameters (variables) $\mathbf{c}_{i}$ defined as follows

$$
\mathbf{c}_{i}=\left[u_{G_{i}}, v_{G_{i}}, u_{W_{i}}, v_{W_{i}}, \Psi_{i}\right]^{\mathrm{T}}
$$

which is consistent with the accepted fifth-class contact kinematic pair model between the wheel and the ground. Dependency (2.5) expresses the location and orientation of the wheel frame $\left\{W_{i}\right\}$ relative to the ground frame $\{G\}$ if the contact variable vector $\mathbf{c}_{i}$ is known.

\subsection{Closed-loop constraints equations of the robot limb}

Relationship (2.5) determines the position of the wheel coordinate system. The wheel frame transformation matrix can be determined, on the other hand, by the $\mathbf{X}$ and robot drives $\boldsymbol{\theta}_{i}$. Transforming the $\{G\}$ frame to the frame of the platform $\{P\}$, followed by the limbs $\left\{L_{i}\right\}$ frame, and then the wheel $\left\{W_{i}\right\}$ coordinate system, gives

$$
{ }^{G} \mathbf{A}_{W_{i}}={ }^{G} \mathbf{A}_{P} \cdot{ }^{P} \mathbf{A}_{L_{i}} \cdot{ }^{L_{i}} \mathbf{A}_{W_{i}}
$$

The first three loop equations take the form

$$
\phi_{1 i, 2 i, 3 i}={ }^{G} \mathbf{p}_{P}+{ }^{G} \mathbf{R}_{P} \cdot\left({ }^{P} \mathbf{p}_{L_{i}}+{ }^{P} \mathbf{R}_{L_{i}} \cdot{ }^{L_{i}} \mathbf{p}_{W_{i}}\right)+{ }^{G} \mathbf{R}_{P} \cdot{ }^{P} \mathbf{R}_{L_{i}} \cdot{ }^{L_{i}} \mathbf{R}_{W_{i}} \cdot \mathbf{f}_{W_{i}}-\mathbf{f}_{G}=\mathbf{0}
$$

The next three equations should be determined from the orientation of contact coordinate systems. The next one results from the condition for the collinearity of the $\widehat{\mathbf{x}}$ or $\widehat{\mathbf{y}}$ of both contact systems $\{C G i\}$ and $\{C W i\}$

$$
\phi_{4 i}=\operatorname{det}\left[\begin{array}{lll}
\mathbf{f}_{u G} & \mathbf{f}_{v G} & { }^{G} \mathbf{R}_{W_{i}} \cdot \mathbf{f}_{u W_{i}}
\end{array}\right]^{\mathrm{T}}=\operatorname{det}\left[\begin{array}{lll}
\mathbf{f}_{u G} & \mathbf{f}_{v G} & { }^{G} \mathbf{R}_{W_{i}} \cdot \mathbf{f}_{v W_{i}}
\end{array}\right]^{\mathrm{T}}=0
$$

The $\widehat{\mathbf{z}}$ axes of both contact systems must be opposite to each other, so their sum gives the zero vector

$$
\widehat{\mathbf{z}}_{C G i}+\widehat{\mathbf{z}}_{C W i}=\mathbf{0} \Longrightarrow \phi_{5 i}=\left\|\left(\mathbf{f}_{u G_{i}} \times \mathbf{f}_{v G_{i}}\right)+{ }^{G} \mathbf{R}_{W_{i}} \cdot\left(\mathbf{f}_{u W_{i}} \times \mathbf{f}_{v W_{i}}\right)\right\|=0
$$


Based on the transformation matrix given by equation (2.4), the rotation matrix by the angle $\Psi_{i}$ between the contact systems $\{C G i\}$ and $\{C W i\}$ can be obtained

$$
\begin{aligned}
& { }^{C G i} \mathbf{R}_{C W i}=\mathbf{R}_{Z}\left(\Psi_{i}\right) \cdot \mathbf{R}_{X}(\pi)={ }^{C G i} \mathbf{R}_{G} \cdot{ }^{G} \mathbf{R}_{C W i} \\
& \quad \Longrightarrow \mathbf{R}_{Z}\left(\Psi_{i}\right)={ }^{C G i} \mathbf{R}_{G} \cdot{ }^{G} \mathbf{R}_{P} \cdot{ }^{P} \mathbf{R}_{L_{i}} \cdot{ }^{{ }_{i}} \mathbf{R}_{W_{i}} \cdot{ }^{W_{i}} \mathbf{R}_{C W i} \cdot \mathbf{R}_{X}(\pi)
\end{aligned}
$$

If constraints (2.8) and (2.9) are met, then the rotation matrix ${ }^{C G i} \mathbf{R}_{C W i}$ will be in the form of a matrix of rotation about axis $\widehat{\mathbf{z}}$, so the last loop equation giving $\Psi_{i}$ angle can be written as:

$$
\phi_{6 i}=\Psi_{i}-\arctan \frac{\sin \Psi_{i}}{\cos \Psi_{i}}=0
$$

where the values of the function $\sin \Psi_{i}$ and $\cos \Psi_{i}$ will be taken from the expressions of the rotation matrix given by equation (2.10). Equations (2.7), (2.8), (2.9) and (2.11) form a system of six closed-loops equations $\phi_{i}$ for one limb of the robot in contact with the ground.

\subsection{Robot platform velocity equations}

To determine robot motion on a defined scene, the derivative of closed-loop contour equations (2.7) for the position of the wheel relative to the ground was derived. Differentiating equation (2.5), the following equation has been obtained

$$
{ }^{G} \mathbf{p}_{W_{i}}=\mathbf{f}_{G}-{ }^{G} \mathbf{R}_{W_{i}} \cdot \mathbf{f}_{W_{i}} \mid \frac{d}{d t} \Longrightarrow{ }^{G} \dot{\mathbf{p}}_{W_{i}}=\dot{\mathbf{f}}_{G}-{ }^{G} \dot{\mathbf{R}}_{W_{i}} \cdot \mathbf{f}_{W_{i}}-{ }^{G} \mathbf{R}_{W_{i}} \cdot \dot{\mathbf{f}}_{W_{i}}
$$

where the expression of the derivative of the ${ }^{j} \dot{\mathbf{R}}_{i}$ rotation matrix can be replaced by the angular velocity matrix $={ }^{j} \boldsymbol{\Omega}_{i}={ }^{j} \dot{\mathbf{R}}_{i} \cdot{ }^{i} \mathbf{R}_{j}$, and the derivative of the point location is the velocity ${ }^{G} \dot{\mathbf{p}}_{W_{i}}={ }^{G} \mathbf{v}_{W_{i}}$.

Transforming this expression to the form ${ }^{j} \dot{\mathbf{R}}_{i}={ }^{j} \boldsymbol{\Omega}_{i} \cdot{ }^{j} \mathbf{R}_{i}$, gives the equation of speed in the form

$$
{ }^{G} \mathbf{v}_{W_{i}}+{ }^{G} \boldsymbol{\Omega}_{W_{i}} \cdot{ }^{G} \mathbf{R}_{W_{i}} \cdot \mathbf{f}_{W_{i}}=\dot{\mathbf{f}}_{G}-{ }^{G} \mathbf{R}_{W_{i}} \cdot \dot{\mathbf{f}} W_{i}=\mathbf{v}_{S L P i}
$$

The left-hand side of the equation represents the velocity of a point on the surface of the wheel that at the given time $t=\tau$ lies at the point of contact $\{C W i\}$ and is permanently located on the surface of the wheel. The velocity $\mathbf{v}_{S L P i}$, therefore, expresses the wheel slip relative to the ground. Differentiation of (2.6) gives both linear $\mathbf{v}_{W_{i}}$ and angular $\boldsymbol{\Omega}_{W_{i}}$ velocities

$$
\begin{aligned}
& { }^{G} \mathbf{v}_{W_{i}}={ }^{G} \mathbf{v}_{P}+{ }^{G} \boldsymbol{\Omega}_{P} \cdot\left({ }^{G} \mathbf{R}_{P} \cdot{ }^{P} \mathbf{p}_{W_{i}}\right)+{ }^{G} \mathbf{R}_{L_{i}} \cdot{ }^{L_{i}} \mathbf{v}_{W_{i}} \\
& { }^{G} \boldsymbol{\Omega}_{W_{i}}={ }^{G} \boldsymbol{\Omega}_{P}+{ }^{G} \mathbf{R}_{L_{i}} \cdot{ }^{L_{i}} \boldsymbol{\Omega}_{W_{i}} \cdot{ }^{L_{i}} \mathbf{R}_{G}
\end{aligned}
$$

Further, by substituting equation (2.14) to the equation for slip velocity (2.13), the following equation has been established

$$
{ }^{G} \mathbf{v}_{S L P i}={ }^{G} \mathbf{v}_{P}+{ }^{G} \boldsymbol{\Omega}_{P} \cdot{ }^{G} \mathbf{p}_{C W i, P}+{ }^{G} \mathbf{R}_{L_{i}} \cdot\left({ }^{L_{i}} \mathbf{v}_{W_{i}}+{ }^{L_{i}} \boldsymbol{\Omega}_{W_{i}} \cdot{ }^{L_{i}} \mathbf{p}_{C W i, W_{i}}\right)
$$

where ${ }^{G} \mathbf{p}_{C W i, P}$ is a vector defined from the location of the platform frame $\{P\}$ to the location of the contact frame $\{C W i\}$ expressed in the global coordinate system $\{G\}$. The condition that the wheel does not slip means that the velocity of the wheel contact point with the ground is zero, $\mathbf{v}_{S L P i}=\mathbf{0}$. This constructs the condition by which the limb drive rates can be determined

$$
{ }^{G} \mathbf{v}_{P}+{ }^{G} \boldsymbol{\Omega}_{P} \cdot{ }^{G} \mathbf{p}_{C W i, P}=-{ }^{G} \mathbf{R}_{L_{i}} \cdot\left({ }^{L_{i}} \mathbf{v}_{W_{i}}+{ }^{L_{i}} \boldsymbol{\Omega}_{W_{i}} \cdot{ }^{L_{i}} \mathbf{p}_{C W i, W_{i}}\right)
$$

By determining the linear and angular velocity of the $\dot{\mathbf{X}}$ platform, the inverse kinematics task can be solved by determining the velocities $\dot{\boldsymbol{\theta}}_{i}$ for each limb separately as in the case of determining the configuration. The path and velocity of the robot platform has to be defined too. 


\subsection{Desired path of the mobile platform}

The movement of the mobile platform is defined along a given flat curve that defines the trajectory of $\mu_{P}$. An additional coordinate system $\left\{P_{0}\right\}$ is defined, which defines the $\pi_{P}$ plane on which the robot platform will move. The $\left\{P_{0}\right\}$ system is fixed relative to the global coordinate system $\left({ }^{G} \mathbf{A}_{P_{0}}=\right.$ const $)$. The robot path is given by the $\mathbf{f}_{P}$ parametric curve depending on one parameter $u_{P}$ (Fig. 5)

$$
\begin{aligned}
& { }_{\mathbf{p}_{P}}={ }^{G} \mathbf{p}_{P_{0}}+{ }^{G} \mathbf{R}_{P_{0}} \cdot \mathbf{f}_{P}\left(u_{P}\right) \\
& \mathbf{f}_{P}\left(u_{P}\right)=\left[f_{x P}\left(u_{P}\right), f_{y P}\left(u_{P}\right), 0\right]^{\mathrm{T}} \\
& \mathbf{f}_{P}: \mathbb{R} \rightarrow \mathbb{R}^{3}
\end{aligned}
$$

The $\mu_{P}$ trajectory of the robot platform determines how the robot moves along the given path relative to time $t$. For this purpose, the robot speeds $G_{\mathbf{v}_{P}}$ and $G_{\boldsymbol{\Omega}_{P}}$ are defined depending on the $u_{P}$ parameter. The linear speed of the robot platform is defined along the tangent vector $\mathbf{t}_{P}$. Differentiating relationship (2.16), we obtain

$$
{ }^{G} \mathbf{v}_{P}={ }^{G} \mathbf{R}_{P_{0}} \cdot \mathbf{t}_{P} \cdot \dot{u}_{P}
$$

where $\mathbf{t}_{P}$ is the tangent vector defining the direction of robot movement

$$
\mathbf{t}_{P}=\mathbf{f}_{u P}=\frac{d \mathbf{f}_{P}}{d u_{P}} \Longrightarrow \widehat{\mathbf{x}}_{P}={ }^{G} \mathbf{R}_{P_{0}} \frac{\mathbf{t}_{P}}{l_{t p}} \quad l_{t p}=\sqrt{\mathbf{t}_{P}^{\mathrm{T}} \cdot \mathbf{t}_{P}}
$$

The orientation of the platform is determined by the axis $\widehat{\mathbf{y}}_{P}$, which is collinear with the normal vector $\mathbf{n}_{P}$ to the path (Fig. 5 ). The vector $\mathbf{n}_{P}$ is determined by rotation of the tangent vector using the following rotation matrix

$$
\mathbf{n}_{P}=\mathbf{R}_{Z}\left(\frac{\pi}{2}\right) \cdot \frac{\mathbf{t}_{P}}{l_{t p}} \Longrightarrow \widehat{\mathbf{y}}_{P}={ }^{G} \mathbf{R}_{P_{0}} \cdot \frac{\mathbf{n}_{P}}{l_{t p}}
$$

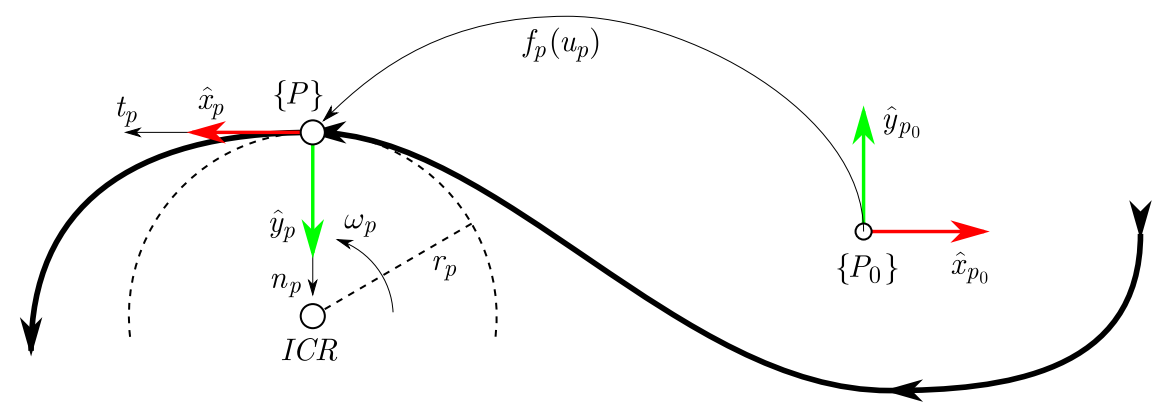

Fig. 5. Sample desired robot trajectory

The robot platform velocity value depends on length of the tangent vector

$$
\left\|G_{\mathbf{v}_{P}}\right\|=l_{t p} \dot{u}_{P} \quad \Longrightarrow \quad \dot{u}_{P}=\frac{v(t)}{l_{t p}}
$$

where $v(t)$ is the time function defining the value of robot speed. The robot movement along the given curve was determined by numerical integration of equation (2.20), resulting in the characteristics of the parameter $u_{P}(t)$.

Equation (2.15), which gives velocities of robot drives, requires that the angular velocity of the platform will be defined. The angular velocity vector is perpendicular to the $\pi_{P}$ plane. The angular velocity matrix $G_{\Omega_{P}}$ is defined by one parameter $\omega_{z p}$ as follows

$$
{ }^{G} \boldsymbol{\Omega}_{P}={ }^{G} \mathbf{R}_{P_{0}} \cdot{ }^{P} \boldsymbol{\Omega}_{P} \cdot{ }^{P_{0}} \mathbf{R}_{G} \quad \text { where } \quad{ }^{P} \boldsymbol{\Omega}_{P}=\omega_{z p}\left[\begin{array}{ccc}
0 & -1 & 0 \\
1 & 0 & 0 \\
0 & 0 & 0
\end{array}\right]
$$


where $\omega_{z p}$ determines length of the platform angular velocity vector depending on the linear velocity ${ }^{G} \mathbf{v}_{P}$ and the radius of curvature $r_{P}$ of a curve (Fig. 6a)

$$
\omega_{z p}=\frac{\left\|{ }^{G} \mathbf{v}_{P}\right\|}{r_{P}}
$$

The radius of curvature $r_{P}$ is the inverse of the curvature of the curve $k_{P}$ determined using derivatives of the parametric function $\mathbf{f}_{P}$

$$
r_{P}=\frac{1}{k_{P}} \quad \text { where } \quad k_{P}=\frac{\left\|\mathbf{f}_{u P} \times \mathbf{f}_{u u P}\right\|}{\left\|\mathbf{f}_{u P}\right\|^{3}}
$$

where $\mathbf{f}_{u u P}$ is the second derivative of the curve parametric function $\mathbf{f}_{P}$ relative to the parameter $u_{P}=d^{2} \mathbf{f}_{P} / d u_{P}^{2}$.

To determine the position and orientation of the platform, the desired trajectory frame $\left\{P_{0}\right\}$ and the curve function $\mathbf{f}_{P}$ must be defined. By specifying the $v(t)$ platform velocity characteristics, the platform velocities needed to simulate the robot motion will be obtained.

\section{Results}

In order to prepare simulation of the robot movement, the ground surface has been defined. It was determined as a spline surface on the basis of control set points. The control point grid was evenly spaced relative to the $\widehat{\mathbf{x}}_{G}$ and $\widehat{\mathbf{y}}_{G}$ axis. The height of control points along $\widehat{\mathbf{z}}_{G}$ axis were chosen randomly in the range between $[-0.2 \mathrm{~m}, 0.2 \mathrm{~m}]$. In this way, the surface of the ground consists of unevenness contained in this range of values (Fig. 7). The surfaces of the wheels were modelled as toruses with a radius equal to the real radius of the robot wheels.

The system defining the given trajectory of the $\left\{P_{0}\right\}$ platform was defined as follows

$$
\left\{P_{0}\right\}:{ }^{G} \mathbf{R}_{P_{0}}=\mathbf{I}_{3} \quad{ }^{G} \mathbf{p}_{P_{0}}=[0,0,0.6 m]^{\mathrm{T}}
$$

In the $\left\{P_{0}\right\}$ system, the platform path was determined using a spline curve made of checkpoints. The checkpoints were defined as in Fig. 5, along straight line segments and the arc with a radius of $1.5 \mathrm{~m}$. Figure $6 \mathrm{~b}$ shows the curvature of the $\mathbf{k}_{P}$ platform path, which was used to determine the $\mu_{I C R}$ trajectory and the instantaneous center of rotation of the $I C R$ platform (Fig. 6a).

A constant robot operational speed $v(t)=0.5 \mathrm{~m} / \mathrm{s}$ was assumed. From relationships $(2.17)$ and (2.21), velocities of the task variables $\dot{\mathbf{X}}$ were obtained. Scripts were built in MATLAB to integrate numerically equations of motion (2.20). In each subsequent step of the integration algorithm, for a given platform location (determined from equation (2.16)) the contact points using contour equations (2.1) where determined. Equations (2.15) gave the remaining velocities

of the robot variables $\dot{\boldsymbol{\theta}}_{i}$. Simulation of 15 seconds of the platform movement was carried out. A visualization of the robot movement is shown in Fig. 7. The red curves determine the trajectories of the contact points of all robot wheels.

Figure 8 presents a graph of displacement of $s_{1}$ rear limbs. The displacement differences of the cylinders in the front limbs were slightly different, shifted in time because the wheels moved along similar paths. From the obtained characteristics, the maximum value of $s_{1 \max }=0.4664 \mathrm{~m}$ and the minimum $s_{1 \mathrm{~min}}=0.3887 \mathrm{~m}$ were determined.

Figure 9 is a graph of the obtained variable velocities $\dot{s}_{1}$ for the rear limbs. As before, they present the key data such as extreme values.

A maximum drive speed of $\dot{s}_{1 \max }=0.1239 \mathrm{~m} / \mathrm{s}$ was obtained. The characteristics shown allow one you to plan the robot path as well as change it due to drive restrictions. 
(a)

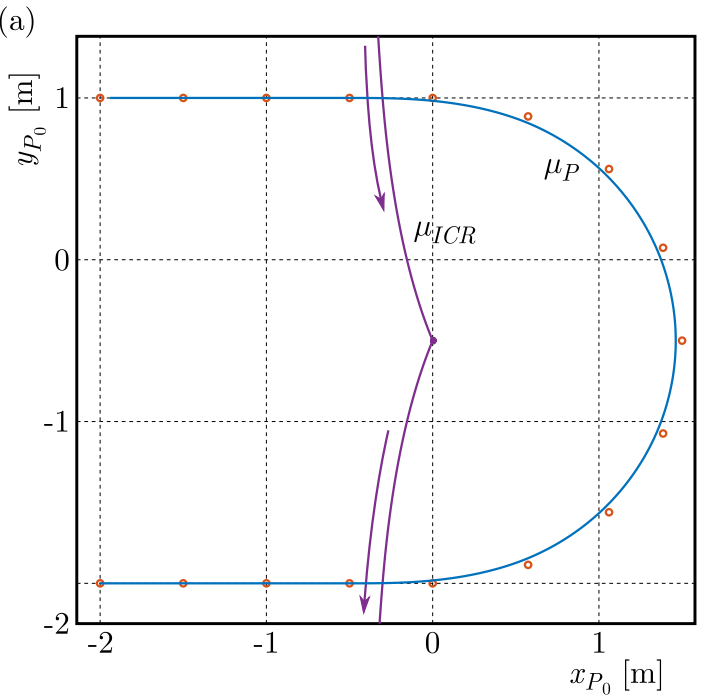

(b)

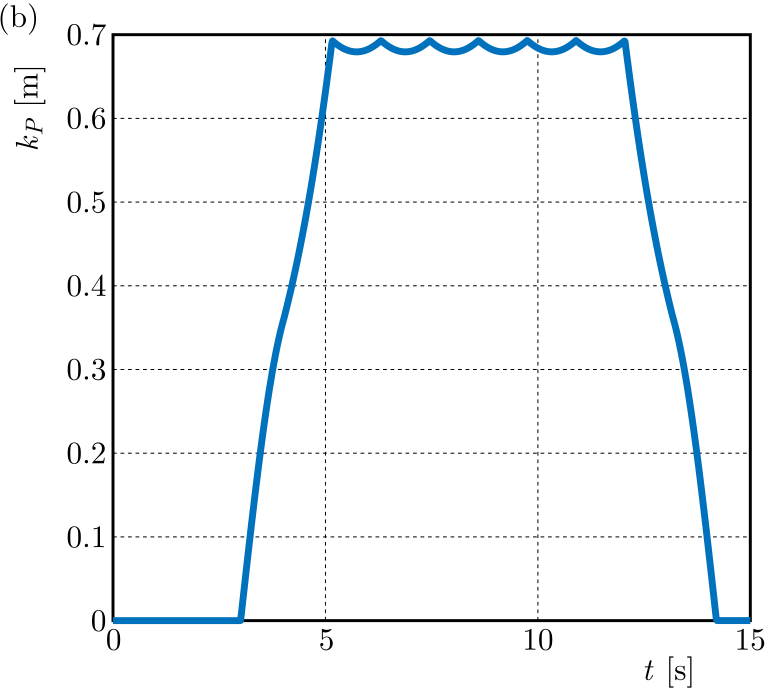

Fig. 6. (a) Specified path and instantaneous centre of rotation of the robot platform, (b) curvature of the platform path

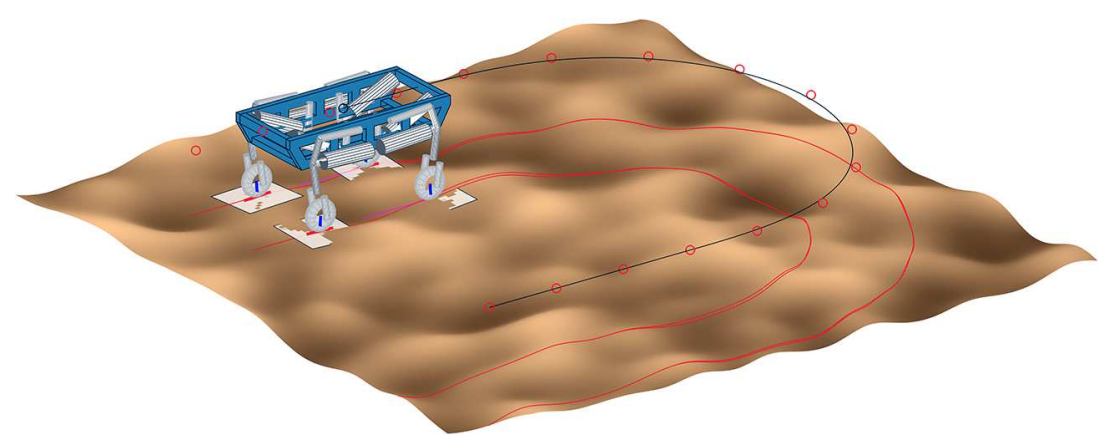

Fig. 7. The result of simulation of motion of a wheeled-legged robot

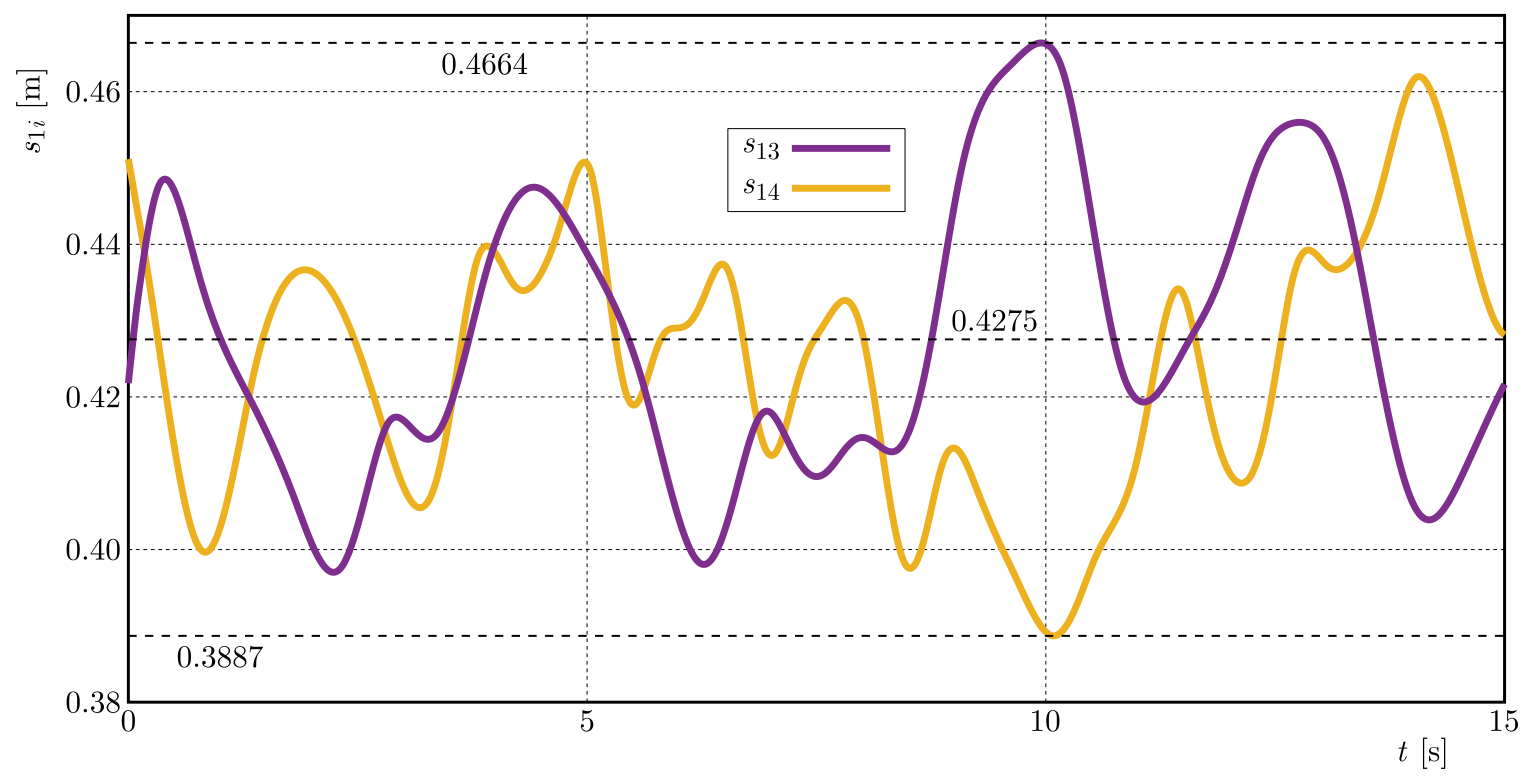

Fig. 8. Runs of the linear actuator displacement 


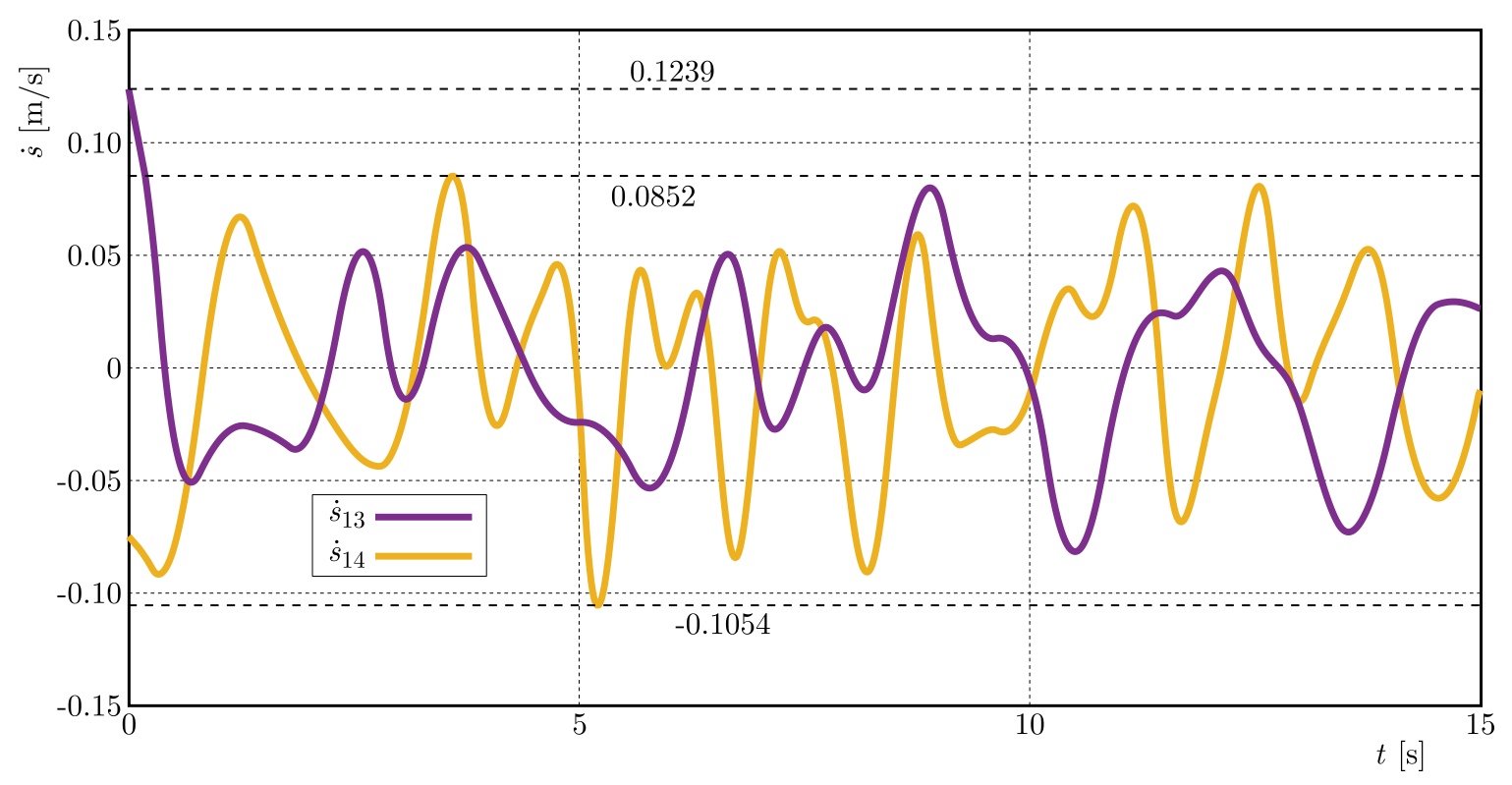

Fig. 9. Runs of the linear actuator velocities

\section{Conclusions}

A robot movement along a given path has been described. Simulation results are kinematic characteristics of robot drives. The presented method allows verification of displacement and velocities of robot actuators. Using the results, the particular robot drives can be chosen during the design process. With the existing prototype, the simulation allows verification of the given trajectory and its possible modification.

The method can be implemented to robot prototyping. A terrain map, in the form of a point cloud, can be obtained using a laser scanner. It was shown that the grid of points can be used to model a smooth ground surface. The simulation results allow one to select such drive velocities to minimize the wheel slip.

\section{References}

1. Avilés S., Rubiano M., Mauledoux M., Valencia C., Jiménez M., 2018, Simulation of a mobile manipulator on webots, International Journal of Online and Biomedical Engineering, 14, $2,90-102$

2. Batchanowski J., 2016, Modelling and simulation studies on the mobile robot with self-leveling chassis, Journal of Theoretical and Applied Mechanics, 54, 1, 149-161, DOI: 10.15632/jtampl.54.1.149

3. Bonnafous D., Lacroix S. Siméon T., 2018, Motion generation for a rover on rough terrains, International Conference on Intelligent Robots and Systems, 2, 784-789, DOI: 10.1109/IROS.2001.976264

4. Chakraborty N., Ghosal A., 2004, Kinematics of wheeled mobile robots on uneven terrain, Mechanism and Machines Theory, 39, 1273-1287

5. Grand C., Benamar F., Plumet F., 2010, Motion kinematics analysis of wheeled-legged rover over 3D surface with posture adaptation, Mechanism and Machine Theory, 45, 3, 477-495

6. https://www.bostondynamics.com/atlas 
7. Novák P., Kot T., Babjak J., Konečný Z., Moczulski W., López Á.R., 2018, Implementation of explosion safety regulations in design of a mobile robot for coal mines, Applied Science, 8, 11, 2300, DOI: 10.3390/app8112300

8. Sperzyński P., Gronowicz A., 2017, Kinematics modelling of mobile robot with articulated limbs without wheel slip, [In:] New Advances in Mechanisms, Mechanical Transmissions and Robotics. Mechanisms and Machine Science, B. Corves, E.-C. Lovasz, M. Hüsing, I. Maniu, C. Gruescu (Eds.), Springer

9. Sperzyński P., Szrek J., 2013, Control of constrained dynamic system of leg of wheel-legged mobile robot, Proceedings of the Second Conference MeTrApp, Bilbao, Spain

10. Townsend J., 2011, ATHLETE mobility performance in long-range traverse, AIAA Space 2011 Conference

11. Ylönen S., HAlme A., 2002, Centaur like service robot, Proceedings of IEEE/RSJ International Conference on Intelligent Robots and Systems, Lausanne Switzerland

12. Wise M., Ferguson M., King D., Dienr W., Dymesich D., 2018, Fetch and Freight: Standard Platforms for Service Robot Applications, Fetch Robotics Inc., San Jose CA, USA

Manuscript received December 2, 2019; accepted for print February 28, 2020 